\title{
Modified Newton's Law of Gravitation due to Minimal Length in Quantum Gravity
}

\author{
Ahmed Farag Ali ${ }^{1}$ and A. Tawfik ${ }^{2,3}$ \\ ${ }^{1}$ Department of Physics, Faculty of Sciences, Benha University, Benha 13518, Egypt \\ ${ }^{2}$ Egyptian Center for Theoretical Physics (ECTP), MTI University, Cairo, Egypt \\ ${ }^{3}$ Research Center for Einstein Physics, Free University of Berlin, 14195 Berlin, Germany \\ Correspondence should be addressed to Ahmed Farag Ali; ahmed.ali@fsc.bu.edu.eg
}

Received 30 November 2012; Accepted 15 January 2013

Academic Editor: George Siopsis

Copyright (C) 2013 A. Farag Ali and A. Tawfik. This is an open access article distributed under the Creative Commons Attribution License, which permits unrestricted use, distribution, and reproduction in any medium, provided the original work is properly cited.

A recent theory about the origin of the gravity suggests that the gravity is originally an entropic force. In this work, we discuss the effects of generalized uncertainty principle (GUP) which is proposed by some approaches to quantum gravity such as string theory, black hole physics, and doubly special relativity theories (DSR), on the area law of the entropy. This leads to a $\sqrt{\operatorname{area}}$-type correction to the area law of entropy which implies that the number of bits $N$ is modified. Therefore, we obtain a modified Newton's law of gravitation. Surprisingly, this modification agrees with different sign with the prediction of Randall-Sundrum II model which contains one uncompactified extra dimension. Furthermore, such modification may have observable consequences at length scales much larger than the Planck scale.

\section{Introduction}

The earliest idea about the connection between gravitation and the existence of a fundamental length was proposed in [1]. In the last two decades, the existence of a minimal length is one of the most interesting predictions of some approaches related to quantum gravity such as string theory, black hole physics, and noncommutative geometry [2-9]. The existence of a minimal length is considered as a consequence of the string theory because strings obviously cannot interact at distances smaller than the string size. Furthermore, the black hole physics suggests that the uncertainty relation should be modified near the Planck energy scale due to the fact that the photons emitted from the black hole suffer from two major errors: the first one is the error by Heisenberg's classical analysis and the second one is because the black hole mass varies during the emission process and the radius of the horizon changes accordingly [2-8]. An interesting measure gedanken experiment was proposed in [10] involving microblack holes at the Planck scale of spacetime which leads to the GUP. This independent model depends on Heisenberg's principle and Schwarzschild radius. Recently, it was found that polymer quantization suggests the existence of minimal length in a similar way to string theory and black hole physics [11]. Therefore, all these different approaches suggest that the standard uncertainty relation in quantum mechanics is modified to yieldgeneralized uncertainty principle (GUP) $[2-8,11]$. In light of this, such modifications can play an essential role as the quantum gravitational corrections which would open an interesting window for quantum gravity phenomenology [1226].

In a one-dimensional chain as the Ising model, when assuming that every single spin is positioned at a distance $d$ apart from the two neighborhoods, the macroscopic state of such a chain is defined by $d$. The entire chain would have various configurations so that if $d \rightarrow l$, the chain has much less configurations than if $d \ll l$, where $l$ is the chain's length. Statistically, the entropy is given by the number of microscopic states $S=k_{B} \ln \Omega$. Due to the second law of thermodynamics, such a system tends to approach a state of maximal entropy so that the chain in the macroscopic state $d$ tends to go to a $d$ state with a much higher entropy. The force that causes such a statistical tendency is defined as the entropic force. In light of this, the entropic force is a 
phenomenological mechanism deriving a system to approach maximum entropy, that is, increasing the number of microscopic states that will be inhered in the system's phase space. There are various examples on the entropic force, for example, polymer molecules and the elasticity of rubber bands.

Recently, Verlinde proposed that the gravity is not a fundamental force and can be considered as an entropic force [27]. The earliest idea about gravity that is regarded as a nonfundamental interaction has been introduced by Sakharov [28-31], where the spacetime background is assumed to emerge as a mean field approximation of underlying microscopic degrees of freedom. Similar behavior is observed in hydrodynamics [32]. It is found that the entropy of black hole is related to the horizon's area at the black hole's horizon, while the temperature is related to the surface gravity. Both entropy and temperature are assumed to be related to the mass of the black hole [33-36]. Thus, the connection between thermodynamics and geometry leads to Einstein's equations of gravitational field from relations connecting heat, entropy, and temperature [37]. Einstein's equations connect energy-momentum tensor with space geometry. Advocating the gravity as nonfundamental interaction leads to the assumption that gravity would be explained as an entropic force caused by changes in the information associated with the positions of material bodies [27]. When combining the entropic force with the Unruh temperature, the second law of Newton is obtained. But when combining it with the holographic principle and using the equipartition law of energy, Newton's law of gravitation is obtained. It was investigated in [38] modification of the entropic force due to corrections to the area law of entropy which is derived from quantum effects and extra dimensions.

Apart from the controversial debate on the origin of gravity $[39,40]$, we investigate the impact of GUP on the entropic force and derive essential quantities including potential modification to Newton's law of gravity.

There were some studies for the effect of some versions of GUP on Newton's law of gravity in [41-44]. Also, noncommutative geometry which is considered as a completely planck scale effect has been studied to derive the modified Newton's law of gravity [45-48]. All these approaches for studying the Planck scale effects on the Newton's law of gravity are based on the following scheme: modified theory of gravity $\rightarrow$ modified black hole entropy $\rightarrow$ modified holographic surface entropy $\rightarrow$ Newton's law corrections. We followed the same scheme in our paper using the new version of GUP proposed in [49-51], and we a got new corrections in our current work, which are distinct from the previous studies. Moreover, we compared our results with Randall-Sundrum model of extra dimension which also predicts the modification of Newton's law of gravity at the Planck scale $[52,53]$, where we think there may be some connection between generalized uncertainty principle and extra dimension theories because they predict similar physics at least for the case of Newton's law of gravity which may be considered as a distinct result from the previous studies.

The present paper is organized as follows. Section 2 reviews briefly the generalized uncertainty principle that was proposed in [49-51]. Section 3 is devoted to review the entropic force and gravitational interaction [27]. The effect of utilizing GUP impact on the entropic force is introduced in Section 4. In Section 5, we estimate the GUP correction to Newton's law of gravitation. The conclusions are given in Section 6.

\section{The Generalized Uncertainty Principle}

It is conjectured that the standard commutation relations at short distances would be modified. A new form of GUP was proposed [49-51] and found consistent with the doubly special relativity (DSR) theories, the string theory, and the black hole physics. It predicts a maximum observable momentum and a minimal measurable length. With satisfying Jacobi identity, GUP is found to ensure the relations $\left[x_{i}, x_{j}\right]=0=$ $\left[p_{i}, p_{j}\right]$ :

$$
\left[x_{i}, p_{j}\right]=i \hbar\left[\delta_{i j}-\alpha\left(p \delta_{i j}+\frac{p_{i} p_{j}}{p}\right)+\alpha^{2}\left(p^{2} \delta_{i j}+3 p_{i} p_{j}\right)\right],
$$

where $\alpha=\alpha_{0} / M_{p} c=\alpha_{0} \ell_{p} / \hbar$ and $M_{p} c^{2}$ stand for Planck energy. $M_{p}$ and $\ell_{p}$ are Planck mass and length, respectively. $\alpha_{0}$ sets on the upper and lower bounds to $\alpha$.

For a particle having an energy scale comparable to Planck's one, the physical momentum would be a subject of modification [49-51]

$$
p_{i}=p_{0 i}\left(1-\alpha p_{0}+2 \alpha^{2} p_{0}^{2}\right)
$$

where $x_{i}=x_{0 i}$ and $p_{0 j}$ satisfy the canonical commutation relations $\left[x_{0 i}, p_{0 j}\right]=i \hbar \delta_{i j}$. Here, $p_{0 i}$ can be interpreted as the momentum at low energies and $p_{i}$ as that at high energies and the variable $p_{0}$ is the value of the momentum at the low energy scales.

This newly proposed GUP suggests that the space is quantized into fundamental units which may be the Planck length. The quantization of space has been shown within the context of loop quantum gravity in [54].

In a series of earlier papers, the effects of GUP were investigated on atomic, condensed matter, preheating phase of the universe systems, black holes at LHC [55-58], the weak equivalence principle (WEP), and the Liouville theorem (LT) in statistical mechanics [59]. It was found that the GUP can potentially explain the small observed violations of the WEP in neutron interferometry experiments [60-62] and also predicts a modified invariant phase space which is relevant to the Liouville theorem. It was derived in [55] the first bound for $\alpha_{0}$ is about $\sim 10^{17}$, which would approximately gives $\alpha \sim 10^{-2} \mathrm{GeV}^{-1}$. The other bound of $\alpha_{0}$ is $\sim 10^{10}$. This bound means that $\alpha \sim 10^{-9} \mathrm{GeV}^{-1}$. As discussed in [63], the exact bound on $\alpha$ can be obtained by comparison with observations and experiments. It seems that the gamma rays burst would allow us to set an upper value for the GUPcharactering parameter $\alpha$ which we would like to report on in the future.

Recently, it has been suggested in [64] that the GUP implications can be measured directly in quantum optics laboratories which definitely confirm the theoretical predictions 
given in $[19,55]$. Definitely, this is considered as a milestone in the road of quantum gravity phenomenology.

In Section 3, we briefly review the assumptions that the gravitational force would originate from an entropic nature.

\section{Gravity as an Entropic Force}

Recently, Verlinde [27] has utilized Sakharov's proposal [2831] that the gravity would not be considered a fundamental force. Concretely, it was suggested that the gravitational force might originate from an entropic nature. As discussed in the introduction, this assumption is based on the relation between the gravitation and thermodynamics [33-36]. According to thermodynamics and holographic principle, Verlinde's approach results in Newton's law. Moreover, the Friedmann equations can also be derived [65]. At temperature $T$, the entropic force $F$ of a gravitational system is given as

$$
F \Delta x=T \Delta S
$$

where $\Delta S$ is the change in the entropy so that at a displacement $\Delta x$ each particle carries its own portion of entropy change. From the correspondence between the entropy change $\Delta S$ and the information about the boundary of the system and using Bekenstein's argument [33-36], it is assumed that $\Delta S=2 \pi k_{B}$, where $\Delta x=\hbar / m$ and $k_{B}$ is the Boltzmann constant:

$$
\Delta S=2 \pi k_{B} \frac{m c}{\hbar} \Delta x
$$

where $m$ is the mass of the elementary component, $c$ is the speed of light, and $\hbar$ is the Planck constant, respectively.

Turning to the holographic principle which assumes that, for any closed surface without worrying about its geometry inside, all physics can be represented by the degrees of freedom on this surface itself. This implies that the quantum gravity can be described by a topological quantum field theory, for which all physical degrees of freedom can be projected onto the boundary [66]. The information about the holographic system is given by $N$ bits forming an ideal gas. It is conjectured that $N$ is proportional to the entropy of the holographic screen:

$$
N=\frac{4 S}{k_{B}}
$$

then, according to Bekenstein's entropy-area relation [33-36]

$$
S=\frac{k_{B} c^{3}}{4 G \hbar} A
$$

Therefore, one gets

$$
N=\frac{A c^{3}}{G \hbar}=\frac{4 \pi r^{2} c^{3}}{G \hbar},
$$

where $r$ is the radius of the gravitational system and the area of the holographic screen $A=4 \pi r^{2}$ is implemented in deriving this equation. It is assumed that each bit emerges outwards from the holographic screen, that is, one dimension. Therefore each bit carries an energy equal to $k_{B} T / 2$, so by using the equipartition rule to calculate the energy of the system, one gets

$$
E=\frac{1}{2} N k_{B} T=\frac{2 \pi c^{3} r^{2}}{G \hbar} k_{B} T=M c^{2}
$$

By substituting (3) and (4) into (8), we get

$$
F=G \frac{M m}{r^{2}}
$$

making it clear that Newton's law of gravitation can be derived from first principles.

In Section 4, we study the effect of GUP approach on the entropic force and hence on the Newton's law of gravitation.

\section{GUP Impact on the Black Hole Thermodynamics}

Taking into consideration the GUP approach [49-51], and because black holes are considered as good laboratories for the clear connection between thermodynamics and gravity, the black hole thermodynamics will be analyzed in this section. Furthermore, how the entropy would be affected will be investigated as well. In Hawking's radiation, the emitted particles are mostly photons and standard model (SM) particles. From kinetic theory of gases, let us assume that gatherings or clouds of points in the velocity space are equally spread in all directions. There is no reason that particles would prefer to be moving in a certain direction. Then, the three moments are simply equal

$$
p_{1} \approx p_{2} \approx p_{3} \text {, }
$$

leading to

$$
\begin{gathered}
p^{2}=\sum_{i=1}^{3} p_{i} p_{i} \approx 3 p_{i}^{2}, \\
\left\langle p_{i}^{2}\right\rangle \approx \frac{1}{3}\left\langle p^{2}\right\rangle .
\end{gathered}
$$

In order to find a relation between $\left\langle p^{2}\right\rangle$ and $\Delta p^{2}$, we assume that the black hole behaves like a black body, while it emits photons. Therefore, from Wien's law, the temperature corresponding to the peak emission is given by

$$
c\langle p\rangle=2.82 T_{H}
$$

We should keep in mind that the numerical factor, 2.82, should be modified by the grey-body factors which arise due to the spacetime curvature around the black hole, but for simplicity we are just ignoring this modification.

From Hawking's uncertainty proposed by Scardigli in [14] and Adler et al. [67], Hawking's temperature reads

$$
T_{H}=\frac{1}{\pi} c \Delta p=\frac{1}{2.82} c\langle p\rangle .
$$


With the relation $\left\langle p^{2}\right\rangle=\Delta p^{2}+\langle p\rangle^{2}$, we get

$$
\begin{gathered}
\langle p\rangle=2.82 \frac{1}{\pi} \Delta p=\sqrt{\mu} \Delta p, \\
\left\langle p^{2}\right\rangle=(1+\mu) \Delta p^{2},
\end{gathered}
$$

where $\mu=(2.82 / \pi)^{2}$. Again, the parameter $\mu$ is modified if we consider the grey-body factors which arise due to the spacetime curvature around the black hole.

In order to have a corresponding inequality for (1), we can utilize the arguments given in $[68,69]$. Then

$$
\Delta x \Delta p \geq \frac{\hbar}{2}\left[1-\alpha\langle p\rangle-\alpha\left\langle\frac{p_{i}^{2}}{p}\right\rangle+\alpha^{2}\left\langle p^{2}\right\rangle+3 \alpha^{2}\left\langle p_{i}^{2}\right\rangle\right] .
$$

It is apparent that implementing the arguments given in (11) and (14) in the inequality given in (15) leads to

$$
\Delta x \Delta p \geq \frac{\hbar}{2}\left[1-\alpha_{0} \ell_{p}\left(\frac{4}{3}\right) \sqrt{\mu} \frac{\Delta p}{\hbar}+2(1+\mu) \alpha_{0}^{2} \ell_{p}^{2} \frac{\Delta p^{2}}{\hbar^{2}}\right] .
$$

The resulting inequality, (16), is the only one that follows from (1). Solving it as a quadratic equation in $\Delta p$ results in

$$
\begin{aligned}
\frac{\Delta p}{\hbar} \geq & \frac{2 \Delta x+\alpha_{0} \ell_{p}((4 / 3) \sqrt{\mu})}{4(1+\mu) \alpha_{0}^{2} \ell_{p}^{2}} \\
& \times\left(1-\sqrt{1-\frac{8(1+\mu) \alpha_{0}^{2} \ell_{p}^{2}}{\left(2 \Delta x+\alpha_{0} \ell_{p}(4 / 3) \sqrt{\mu}\right)^{2}}}\right) .
\end{aligned}
$$

The negatively signed solution is considered as the one that refers to the standard uncertainty relation as $\ell_{p} / \Delta x \rightarrow 0$. Using the Taylor expansion, we obviously find that

$$
\Delta p \geq \frac{1}{\Delta x}\left(1-\frac{2}{3} \alpha_{0} \ell_{p} \sqrt{\mu} \frac{1}{\Delta x}\right) .
$$

Because the energy change reads $\Delta E \approx c \Delta p$ and according to Scardigli in [14] and Adler et al. [67], one can define the uncertainty in the energy $\Delta E$ as the energy carried away from the black hole through the emitted photon. In the following, we implement the procedure introduced in $[70,71]$. We utilize the GUP approach [49-51] together with the assumptions given in (11) and (14). Then, using natural units that $\hbar=c=1$

$$
\Delta E \geq \frac{1}{\Delta x}\left(1-\frac{2}{3} \alpha_{0} \ell_{p} \sqrt{\mu} \frac{1}{\Delta x}\right) .
$$

For a black hole absorbing a quantum particle with energy $E$ and size $R$, the area of the black hole is supposed to increase by the amount [33]:

$$
\Delta A \geq 8 \pi \ell_{p}^{2} E R
$$

The quantum particle itself implies the existence of finite bound given by

$$
\Delta A_{\min } \geq 8 \pi \ell_{p}^{2} E \Delta x
$$

Using (19) in the inequality (21), we obtain

$$
\Delta A_{\min } \geq 8 \pi \ell_{p}^{2}\left[1-\frac{2}{3} \alpha_{0} \ell_{p} \sqrt{\mu} \frac{1}{\Delta x}\right]
$$

According to the argument given in $[70,71]$, the length scale is chosen to be the inverse surface gravity

$$
\Delta x=2 r_{s}
$$

where $r_{s}$ is the Schwarzschild radius. This argument implies that

$$
(\Delta x)^{2} \sim \frac{A}{\pi} .
$$

Substituting (24) into (22), we got

$$
\Delta A_{\min }=\lambda \ell_{p}^{2}\left[1-\frac{2}{3} \alpha_{0} \ell_{p} \sqrt{\frac{\mu \pi}{A}}\right]
$$

where parameter $\lambda$ will be fixed later. According to [33$36]$, the black hole's entropy is conjectured to depend on the horizon's area. From the information theory [72], it has been found that the minimal increase of entropy should be independent of the area. It is just one "bit" of information which is $b=\ln (2)$,

$$
\frac{d S}{d A}=\frac{\Delta S_{\min }}{\Delta A_{\min }}=\frac{b}{\lambda \ell_{p}^{2}\left[1-(2 / 3) \alpha_{0} \ell_{p} \sqrt{\mu \pi A}\right]},
$$

where $b$ is a parameter. By expanding the last expression in orders of $\alpha$ and then integrating it, we get the entropy

$$
S=\frac{b}{\lambda \ell_{p}^{2}}\left[A+\frac{4}{3} \alpha_{0} \ell_{p} \sqrt{\mu \pi A}\right]
$$

Using the Hawking-Bekenstein assumption, which relates entropy with the area, the value of constants $b / \lambda=1 / 4$, so that

$$
S=\frac{A}{4 \ell_{p}^{2}}+\frac{2}{3} \alpha_{0} \sqrt{\pi \mu \frac{A}{4 \ell_{p}^{2}}}
$$

Although it was found in [73] that the power-law corrections to Bekenstein-Hawking area entropy are ruled out based on arguments from the Boltzmann-Einstein formula, it was found that the power-law corrections may explain the observed cosmic acceleration today [74].

We conclude that the entropy is directly related to the area and gets a correction when applying GUP approach. The temperature of the black hole is

$$
T=\frac{\kappa}{8 \pi} \frac{d A}{d S}=\frac{\kappa}{8 \pi}\left[1-\frac{2}{3} \alpha_{0} \ell_{p} \sqrt{\mu \frac{\pi}{A}}\right]
$$

So far, we conclude that not only the temperature is proportional to the surface gravity but also it depends on the black hole's area. 


\section{Modified Newton's Law of Gravitation due to GUP}

In this section we study the implications of the corrections calculated for the entropy in (28) and calculate how the number of bits of (5) would be modified which assume new corrections to Newton's law of gravitation. Using the corrected entropy given in (28), we find that the number of bits should also be corrected as follows:

$$
N^{\prime}=\frac{4 S}{k_{B}}=\frac{A}{\ell_{p}^{2}}+\frac{4}{3} \alpha_{0} \sqrt{\mu \pi \frac{A}{\ell_{p}^{2}}} .
$$

By substituting (30) into (8) and using (3), we get

$$
E=F c^{2}\left(\frac{r^{2}}{m G}+\frac{\alpha \sqrt{\mu} r}{3 m G}\right) .
$$

It is apparent that (31) implies a modification in Newton's law of gravitation

$$
F=G \frac{M m}{r^{2}}\left(1-\frac{\alpha \sqrt{\mu}}{3 r}\right) .
$$

This equation states that the modification in Newton's law of gravity seems to agree with the predictions of RandallSundrum II model [52] which contains one uncompactified extra dimension and length scale $\Lambda_{R}$. The only difference is the sign. The modification in Newton's gravitational potential on brane [53] is given as

$$
V_{R S}= \begin{cases}-G \frac{m M}{r}\left(1+\frac{4 \Lambda_{R}}{3 \pi r}\right), & r \ll \Lambda_{R}, \\ -G \frac{m M}{r}\left(1+\frac{2 \Lambda_{R}}{3 r^{2}}\right), & r \gg \Lambda_{R},\end{cases}
$$

where $r$ and $\Lambda_{R}$ are the radius and the characteristic length scale, respectively. It is clear that the gravitational potential is modified at short distance. We notice that our result, (32), agrees with different sign with (33) when $r \ll \Lambda_{R}$. This result would say that $\alpha \sim \Lambda_{R}$ which would help set a new upper bound on the value of the parameter $\alpha$. This means that the proposed GUP approach $[49,51]$ is apparently able to predict the same physics as Randall-Sundrum II model. The latter assumes the existence of one extra dimension compactified on a circle whose upper and lower halves are identified. If the extra dimensions are accessible only to gravity and not to the standard model field, the bound on their size can be fixed by an experimental test of Newton's law of gravitation, which has only been led down to $\sim 4$ millimeter. This was the result, about ten years ago [75]. In recent gravitational experiments, it is found that the Newtonian gravitational force, the $1 / r^{2}$-law, seems to be maintained up to $\sim 0.13-$ $0.16 \mathrm{~mm}[76,77]$. However, it is unknown whether this law is violated or not at sub- $\mu \mathrm{m}$ range. Further implications of this modifications have been discussed in [78] which could be the same for the GUP modification which is calculated in this paper. This similarity between the GUP implications and extra dimensions implications would assume new bounds on the GUP parameter $\alpha$ with respect to the extra dimension length scale $\Lambda_{R}$.

\section{Conclusions}

In this paper, we tackle the consequences of the quantum gravity on the entropic force approach which assumes a new origin of the gravitational force. We found that the quantum gravity corrections lead to a modification in the area law of the entropy which leads to a modification in the number of bits N. According to Verlinde's theory of entropic force, Newton's law of gravitation would acquire new quantum gravity corrections due to the modified number of bits. The modification in Newton's Law of gravitation surprisingly agrees with the corrections predicted by Randall-Sundrum II model with different sign. This would open a new naturally arising question in our proposed research if the GUP and extra dimensions theories would predict the same physics. We hope to report on this in the future.

\section{Acknowledgments}

The research of A. F. Ali is supported by Benha University. The research of AT has been partly supported by the GermanEgyptian Scientific Projects (GESP ID: 1378). A. F. Ali and A. N. Tawfik would like to thank Professor Antonino Zichichi for his kind invitation to attend the International School of Subnuclear Physics 2012 at the "Ettore Majorana Foundation and Centre for Scientific Culture" in Erice, Italy, where the present work was started. The authors gratefully thank the anonymous referee for useful comments and suggestions which helped to improve the paper.

\section{References}

[1] C. A. Mead, "Possible connection between gravitation and fundamental length," Physical Review, vol. 135, pp. B849-B862, 1964.

[2] D. Amati, M. Ciafaloni, and G. Veneziano, "Can spacetime be probed below the string size?” Physics Letters B, vol. 216, no. 1-2, pp. 41-47, 1989.

[3] M. Maggiore, "A generalized uncertainty principle in quantum gravity," Physics Letters B, vol. 304, no. 1-2, pp. 65-69, 1993.

[4] M. Maggiore, "Quantum groups, gravity, and the generalized uncertainty principle," Physical Review D, vol. 49, no. 10, pp. 5182-5187, 1994.

[5] M. Maggiore, "The algebraic structure of the generalized uncertainty principle," Physics Letters B, vol. 319, no. 1-3, pp. 83-86, 1993.

[6] L. J. Garay, "Quantum gravity and minimum length," International Journal of Modern Physics A, vol. 10, pp. 145-166, 1995.

[7] S. Hossenfelder, M. Bleicher, S. Hofmann, J. Ruppert, S. Scherer, and H. Stoecker, "Signatures in the Planck regime," Physics Letters B, vol. 575, no. 1-2, pp. 85-99, 2003.

[8] C. Bambi and F. R. Urban, "Natural extension of the generalized uncertainty principle," Classical and Quantum Gravity, vol. 25, Article ID 095006, 2008.

[9] G. Amelino-Camelia, G. Gubitosi, and F. Mercati, "Discreteness of area in noncommutative space," Physics Letters B, vol. 676, no. 4-5, pp. 180-183, 2009.

[10] F. Scardigli, "Generalized uncertainty principle in quantum gravity from micro-black hole gedanken experiment," Physics Letters B, vol. 452, no. 1-2, pp. 39-44, 1999. 
[11] G. M. Hossain, V. Husain, and S. S. Seahra, "Backgroundindependent quantization and the uncertainty principle," Classical and Quantum Gravity, vol. 27, no. 16, Article ID 165013, 8 pages, 2010.

[12] K. Nozari and B. Fazlpour, "Generalized uncertainty principle, modified dispersion relations and the early universe thermodynamics," General Relativity and Gravitation, vol. 38, no. 11, pp. 1661-1679, 2006.

[13] R. J. Adler and D. I. Santiago, "On gravity and the uncertainty principle," Modern Physics Letters A, vol. 14, no. 20, article 1371, 1999.

[14] F. Scardigli, "Some heuristic semi-classical derivations of the Planck length, the Hawking effect and the Unruh effect," Nuovo Cimento B, vol. 110, no. 9, pp. 1029-1034, 1995.

[15] A. Kempf, G. Mangano, and R. B. Mann, "Hilbert space representation of the minimal length uncertainty relation," Physical Review D, vol. 52, no. 2, pp. 1108-1118, 1995.

[16] S. Hossenfelder, "Interpretation of quantum field theories with a minimal length scale," Physical Review D, vol. 73, no. 10, Article ID 105013, 9 pages, 2006.

[17] C. Bambi, "A revision of the generalized uncertainty principle," Classical and Quantum Gravity, vol. 25, no. 10, Article ID 105003, 9 pages, 2008.

[18] J. Y. Bang and M. S. Berger, "Quantum mechanics and the generalized uncertainty principle," Physical Review D, vol. 74, no. 12, Article ID 125012, 8 pages, 2006.

[19] S. Das and E. C. Vagenas, "Universality of quantum gravity corrections," Physical Review Letters, vol. 101, no. 22, Article ID 221301, 4 pages, 2008.

[20] S. Das and E. C. Vagenas, "Phenomenological implications of the generalized uncertainty principle," Canadian Journal of Physics, vol. 87, no. 3, pp. 233-240, 2009.

[21] S. Hossenfelder, "Minimal length scale scenarios for quantum gravity," Living Reviews in Relativity, vol. 16, article 2, 2013.

[22] A. Kempf, "Non-pointlike particles in harmonic oscillators," Journal of Physics A, vol. 30, no. 6, pp. 2093-2101, 1997.

[23] F. Brau, "Minimal length uncertainty relation and the hydrogen atom," Journal of Physics A, vol. 32, no. 44, pp. 7691-7696, 1999.

[24] M. Sprenger, P. Nicolini, and M. Bleicher, "Physics on smallest scales-an introduction to minimal length phenomenology," European Journal of Physics, vol. 33, no. 4, pp. 853-862, 2012.

[25] M. Sprenger, P. Nicolini, and M. Bleicher, "Neutrino oscillations as a novel probe for a minimal length," Classical and Quantum Gravity, vol. 28, no. 23, Article ID 235019, 2011.

[26] J. Mureika, P. Nicolini, and E. Spallucci, "Could any black holes be produced at the LHC?" Physical Review D, vol. 85, no. 10, Article ID 106007, 8 pages, 2012.

[27] E. P. Verlinde, "On the origin of gravity and the laws of Newton," Journal of High Energy Physics, vol. 2011, article 29, 2011.

[28] A. D. Sakharov, "Vacuum quantum fluctuations in curved space and the theory of gravitation," Soviet Physics, vol. 12, no. 1, pp. 1040-1041, 1968.

[29] A. D. Sakharov, "Vacuum quantum fluctuations in curved space and the theory of gravitation," Doklady Akademii Nauk SSSR, vol. 177, no. 1, pp. 70-71, 1967.

[30] A. D. Sakharov, "Vacuum quantum fluctuations in curved space and the theory of gravitation," Soviet Physics Uspekhi, vol. 34, no. 5, article 394, 1991.

[31] A. D. Sakharov, "Vacuum quantum fluctuations in curved space and the theory of gravitation," General Relativity and Gravitation, vol. 32, no. 2, article 365, 2000.
[32] M. Visser, "Sakharov's induced gravity: a modern perspective," Modern Physics Letters A, vol. 17, no. 15-17, pp. 977-991, 2002.

[33] J. D. Bekenstein, "Black holes and entropy," Physical Review D, vol. 7, pp. 2333-2346, 1973.

[34] J. M. Bardeen, B. Carter, and S. W. Hawking, "The four laws of black hole mechanics," Communications in Mathematical Physics, vol. 31, no. 2, pp. 161-170, 1973.

[35] S. W. Hawking, "Particle creation by black holes," Communications in Mathematical Physics, vol. 43, no. 3, pp. 199-220, 1975.

[36] S. W. Hawking, "Erratum: particle creation by black holes, Communications in Mathematical Physics, 43, 3, 199-220, 1975," Communications in Mathematical Physics, vol. 46, no. 2, article 206, 1976.

[37] T. Jacobson, "Thermodynamics of spacetime: the Einstein equation of state," Physical Review Letters, vol. 75, no. 7, pp. 1260-1263, 1995.

[38] Y. Zhang, Y. Gong, and Z. -H. Zhu, "Modified gravity emerging from thermodynamics and holographic principle," International Journal of Modern Physics D, vol. 20, no. 8, pp. 1505-1519, 2011.

[39] A. Kobakhidze, "Gravity is not an entropic force," Physical Review D, vol. 83, no. 2, Article ID 021502, 3 pages, 2011.

[40] S. Hossenfelder, "Comments on and comments on comments on Verlinde's paper 'on the origin of gravity and the laws of Newton," http://arxiv.org/abs/1003.1015.

[41] K. Nozari, P. Pedram, and M. Molkara, "Minimal length, maximal momentum and the entropic force law," International Journal of Theoretical Physics, vol. 51, no. 4, pp. 1268-1275, 2012.

[42] P. Nicolini, "Nonlocal and generalized uncertainty principle black holes," http://arxiv.org/abs/1202.2102.

[43] S. Ghosh, "Planck scale effect in the entropic force law," Modern Physics Letters A, http://arxiv.org/abs/1003.0285.

[44] M. A. Santos and I. V. Vancea, "Entropic law of force, emergent gravity and the uncertainty principle," Modern Physics Letters A, vol. 27, no. 2, Article ID 1250012, 11 pages, 2012.

[45] P. Nicolini, "Entropic force, noncommutative gravity, and ungravity," Physical Review D, vol. 82, no. 4, Article ID 044030, 8 pages, 2010.

[46] C. Bastos, O. Bertolami, N. C. Dias, and J. N. Prata, "Entropic gravity, phase-space noncommutativity and the equivalence principle," Classical and Quantum Gravity, vol. 28, no. 12, Article ID 125007, 8 pages, 2011.

[47] K. Nozari and S. Akhshabi, "Noncommutative geometry inspired entropic inflation," Physics Letters B, vol. 700, no. 2, pp. 91-96, 2011.

[48] S. H. Mehdipour, A. Keshavarz, and A. Keshavarz, "Entropic force approach in a noncommutative charged black hole and the equivalence principle," Europhysics Letters, vol. 98, no. 1, Article ID 10002, 2012.

[49] A. F. Ali, S. Das, and E. C. Vagenas, "Discreteness of space from the generalized uncertainty principle," Physics Letters B, vol. 678, no. 5, pp. 497-499, 2009.

[50] A. F. Ali, S. Das, and E. C. Vagenas, "The generalized uncertainty principle and quantum gravity phenomenology," in 12th Marcel Grossmann Meeting on General Relativity (MG 12), pp. 24072409, Paris, France, July 2009.

[51] S. Das, E. C. Vagenas, and A. F. Ali, "Discreteness of space from GUP II: relativistic wave equations," Physics Letters B, vol. 690, no. 4, pp. 407-412, 2010. 
[52] L. Randall and R. Sundrum, "An alternative to compactification," Physical Review Letters, vol. 83, no. 23, pp. 4690-4693, 1999.

[53] P. Callin and F. Ravndal, "Higher order corrections to the Newtonian potential in the Randall-Sundrum model," Physical Review D, vol. 70, no. 10, Article ID 104009, 12 pages, 2004.

[54] T. Thiemann, "A length operator for canonical quantum gravity," Journal of Mathematical Physics, vol. 39, no. 6, pp. 3372-3392, 1998.

[55] A. F. Ali, S. Das, and E. C. Vagenas, "Proposal for testing quantum gravity in the lab," Physical Review D, vol. 84 , no. 4 , Article ID 044013, 10 pages, 2011.

[56] W. Chemissany, S. Das, A. F. Ali, and E. C. Vagenas, "Effect of the Generalized Uncertainty Principle on post-inflation preheating," Journal of Cosmology and Astroparticle Physics, vol. 2011, article 017, 2011.

[57] A. F. Ali, "No existence of black holes at LHC due to minimal length in quantum gravity," Journal of High Energy Physics, vol. 2012, article 67, 2012.

[58] A. F. Ali, H. Nafie, and M. Shalaby, "Minimal length, maximal energy and black-hole remnants," Europhysics Letters, vol. 100, no. 2, Article ID 20004, 2012.

[59] A. F. Ali, "Minimal length in quantum gravity, equivalence principle and holographic entropy bound," Classical and Quantum Gravity, vol. 28, no. 6, Article ID 065013, 2011.

[60] R. Collela, A. W. Overhauser, and S. A. Werner, "Observation of gravitationally induced quantum interference," Physical Review Letters, vol. 34, no. 23, pp. 1472-1474, 1975.

[61] K. C. Littrell, B. E. Allman, and S. A. Werner, "Two-wavelengthdifference measurement of gravitationally induced quantum interference phases," Physical Review A, vol. 56, no. 3, pp. 17671780, 1997.

[62] A. Camacho and A. Camacho-Galvan, "Test of some fundamental principles in physics via quantum interference with neutrons and photons," Reports on Progress in Physics, vol. 70, pp. 1-56, 2007, http://arxiv.org/abs/0810.1325.

[63] A. Tawfik, H. Magdy, and A. Farag Ali, "Lorentz invariance violation and generalized uncertainty principle," http://arxiv.org/ abs/1205.5998.

[64] I. Pikovski, M. R. Vanner, M. Aspelmeyer et al., "Probing Planck-scale physics with quantum optics," Nature Physics, vol. 8, no. 5, pp. 393-397, 2012.

[65] R.-G. Cai, L.-M. Cao, and N. Ohta, "Friedmann equations from entropic force," Physical Review D, vol. 81, no. 6, Article ID 061501, 4 pages, 2010.

[66] G. t'Hooft, "Dimensional reduction in quantum gravity," http:// arxiv.org/abs/gr-qc/9310026.

[67] R. J. Adler, P. Chen, and D. I. Santiago, "The generalized uncertainty principle and black hole remnants," General Relativity and Gravitation, vol. 33, no. 12, pp. 2101-2108, 2001.

[68] M. Cavaglià, S. Das, and R. Maartens, "Will we observe black holes at the LHC?” Classical and Quantum Gravity, vol. 20, no. 15, pp. L205-L212, 2003.

[69] M. Cavaglia and S. Das, "How classical are TeV-scale black holes?" Classical and Quantum Gravity, vol. 21, no. 19, pp. 45114522, 2004.

[70] A. J. M. Medved and E. C. Vagenas, "When conceptual worlds collide: the generalized uncertainty principle and the Bekenstein-Hawking entropy," Physical Review D, vol. 70, no. 12, Article ID 124021, 5 pages, 2004.
[71] B. Majumder, "Black hole entropy and the modified uncertainty principle: a heuristic analysis," Physics Letters B, vol. 703, no. 4, pp. 402-405, 2011.

[72] C. Adami, "The physics of information," http://arxiv.org/abs/ quant-ph/0405005.

[73] S. Hod, "High-order corrections to the entropy and area of quantum black holes," Classical and Quantum Gravity, vol. 21, no. 14, pp. L97-L100, 2004.

[74] P. Wang, "Horizon entropy in modified gravity," Physical Review D, vol. 72, no. 2, Article ID 024030, 4 pages, 2005.

[75] C. D. Hoyle, U. Schmidt, B. R. Heckel et al., "Submillimeter tests of the gravitational inverse square law: a search for "large" extra dimensions," Physical Review Letters, vol. 86, no. 8, pp. 14181421, 2001.

[76] S.-Q. Yang, B.-F. Zhan, Q.-L. Wang et al., "Test of the gravitational inverse square law at millimeter ranges," Physical Review Letters, vol. 108, no. 8, Article ID 081101, 5 pages, 2012.

[77] C. D. Hoyle, D. J. Kapner, B. R. Heckel et al., "Submillimeter tests of the gravitational inverse-square law," Physical Review D, vol. 70, no. 4, Article ID 042004, 31 pages, 2004.

[78] F. Buisseret, B. Silvestre-Brac, and V. Mathieu, "Modified Newton's law, braneworlds, and the gravitational quantum well," Classical and Quantum Gravity, vol. 24, no. 4, pp. 855-865, 2007. 

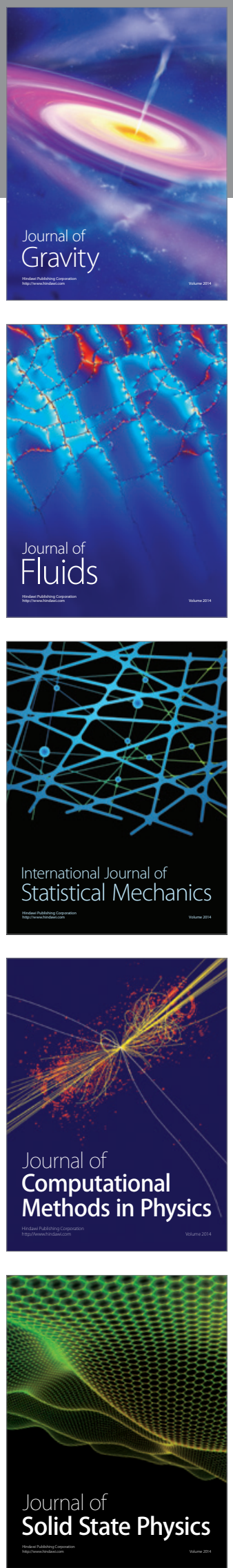

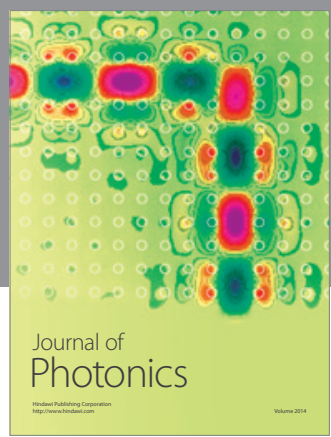

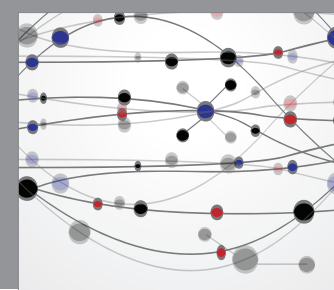

The Scientific World Journal

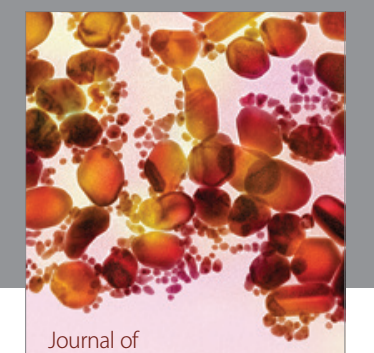

Soft Matter
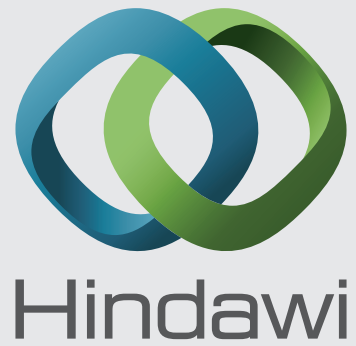

Submit your manuscripts at

http://www.hindawi.com
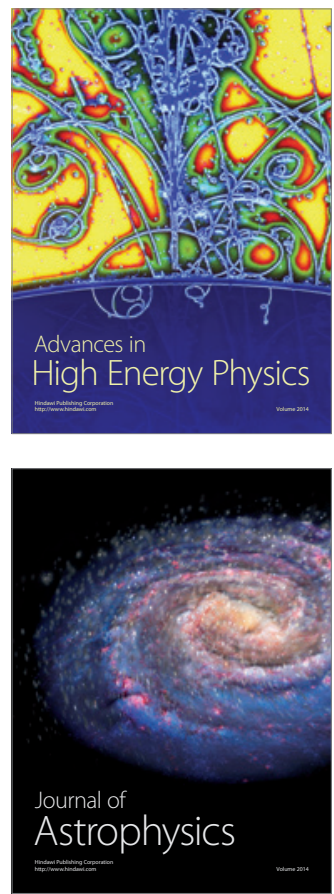
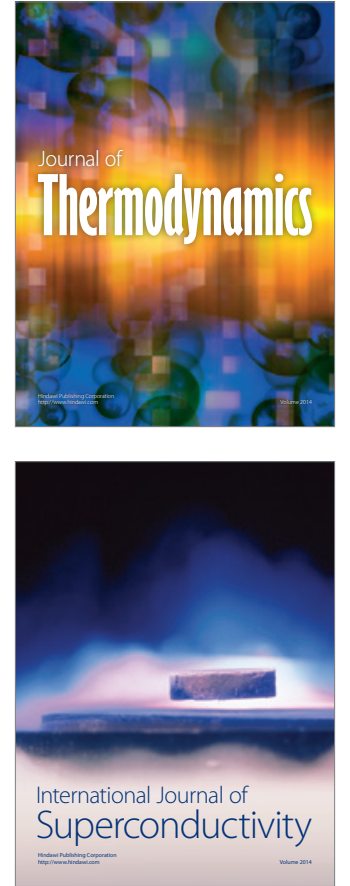
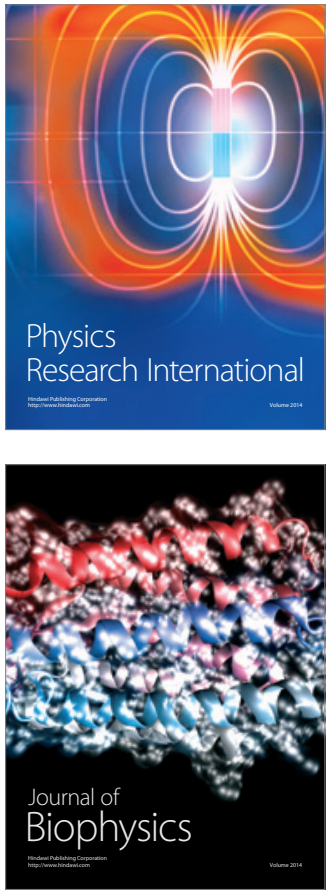
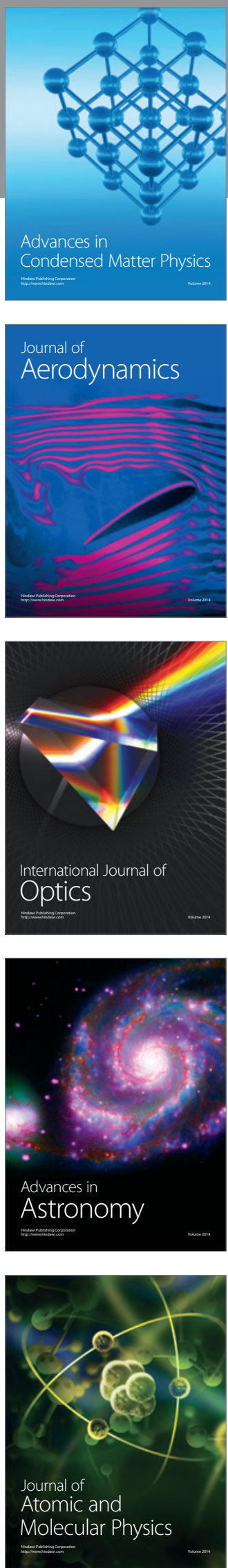